\title{
A mathematical model of stress reaction: Individual differences in threshold and duration
}

\author{
DANKA SAVIĆ \\ Vinča Institute of Nuclear Sciences, Belgrade, Yugoslavia \\ and \\ GORAN KNEŽEVIĆ and GORAN OPAČIĆ \\ University of Belgrade, Belgrade, Yugoslavia
}

\begin{abstract}
People differ in what they experience as stressful and to what extent. We define a variable-stress threshold $(\sigma)$ - that links the hypothalamo-pituitary-adrenocortical (HPA) axis and the memory system in a feedback mechanism. Current $\sigma$ dictates the intensity of a stimulus that turns the stress response on. On the other hand, each "jump" of the HPA axis helps long-term registration of the stressful event via concentration changes of some of its products, consequently changing the value of $\sigma$ for future stressors. After the action of a strong exterior stressor, the new stressful memory acts as an internal source of stress. We assume that its intensity decreases with the rate of processing the stressful information. This process is characterized by a time parameter $\tau$. Both $\sigma$ and $\tau$ are individual: They depend on personality traits, genetic as well as acquired. The mathematical model presented here simulates the feedback mechanism between the HPA axis and the memory system involved in stress reaction.
\end{abstract}

The classic definition of stress originates from Hans Selye. According to him, stress comprises a set of unspecific reactions of an organism that are activated to maintain homeostasis in changed environmental conditions (i.e., to help the organism adapt to them). Stimuli that provoke stress are called stressors, and, accordingly, complex situations that lead to stress are qualified as stressful.

Dienstbier (1989) makes a distinction between the types of stressors (and, accordingly, of stress) that challenge and those that cause threat/harm/loss. This categorization is important because it assumes different underlying mechanisms and the involvement of different neuroendocrine systems. It is well known that two neuroendocrine systems are mainly involved in stress responses: the symphatetic-nervous-system-stimulated catecholaminic (adrenal-medullary) system and the hypothalamopituitary-adrenocortical (HPA) axis. Dienstbier links the challenge predominantly to the first system, whereas the threat/harm/ loss additionally turns on the HPA axis. Our use of the terms stress and stressful will assume the involvement of the HPA axis.

People react differently in stressful situations. What is stressful for one does not have to be stressful for another person, or not to the same extent. Empirical studies show

The authors thank Rachel Yehuda for taking an interest in the present work and for useful advice, Vladimir Jovic for sharing his professional experiences on traumatized patients and for providing a clearer picture of stress reaction, and Scott Poethig for language corrections. Correspondence should be addressed to D. Savić, Vinča Institute of Nuclear Sciences, Laboratory for Theoretical Physics and Condensed Matter Physics (020/2), P. O. Box 522, Belgrade 11001, Yugoslavia (e-mail: savicana@eunet.yu). that level of distress caused by the same stressor is different for different people (Cox \& Ferguson, 1991). To express these differences, we introduce the parameter $\sigma$, a threshold for psychological stressors that can be defined as a minimal degree of external stimulus able to evoke HPA axis arousal. The following is a statistical definition: For any given situation, a stress threshold is an inverse measure of probability of experiencing it as stressful. This means that if there is a large number of situations and if two people with different thresholds go through all of them, a vulnerable individual will experience more of these situations as stressful than will a tough person, with inverse proportion to their thresholds. At the same time, this formulation allows a particular situation to be stressful for a tougher person (higher $\sigma$ ) and not stressful for the more vulnerable one (lower $\sigma$ ). In other words, anyone can have conditioned stressors that are specific to him/ her even if he/she is generally tough.

We can ask further, What causes individual differences in recognizing a situation as stressful? Generally, there are two groups of factors determining stress threshold genetic and experiential. These factors mutually influence each other, thus changing stress vulnerability with accumulation of life experience. The core of vulnerability is a wide generalized disposition known as neuroticismone of the five basic personality dimensions, according to the "big five" model of personality structure and functioning (Costa \& McCrae, 1992). Costa and McCrae define it as a "tendency to experience negative affects such as fear, sadness, embarrassment, anger, guilt and disgust." According to these authors, main indicators of neuroticism are anxiety, angry hostility, depression, selfconsciousness, impulsiveness, and vulnerability. This 
fundamental dimension of human behavior regularly appears under the same name or similar names in all major, solidly operationalized, personality theories. Janet (1909; according to van der Kolk \& van der Hart, 1989) labels it vulnerability, Eysenck and Eysenck (1969) label it neuroticism, Momirovic, Wolf, and Dzamonja (1992) label it general conative dysfunction, Cloninger (1987) labels it harm avoidance, Guilford (1959) labels it the negative pole of emotional stability, Cattell (1970) labels it anxiety, Tellegen et al. (1988) label it negative emotionality, and so on.

Despite the fact that increase of vulnerability is a general reaction starting right after a traumatic event, the longitudinal consequences of such an event can be different: Some people come out toughened by this experience, and some become more vulnerable. To cite McFarlane and Yehuda (1996): "The experience of such events can modify an individual's vulnerability to subsequent traumatic events ... Equally, such experiences can become powerful sources of motivation for some individuals, indicating that trauma can have positive effects on those who survive the ordeal ..."

Accumulating life experience means learning. When a person is being exposed to a stressor, he/she reacts in a certain way. There are clinical observations suggesting that the first days after a traumatic event are when people evaluate their behavior (Shalev, 1996). This self-evaluation is very important for the final stress effect. If coping is evaluated as successful, a subsequent encounter of this type of situation will be less stressful, and the contrary. Coping skills themselves also strongly depend on personality traits-mainly, neuroticism. Without an intervention from the environment, a highly neurotic (vulnerable) individual is likely to fall into a baleful feedback loop: high neuroticism leads to poor coping, which leads to negative self-evaluation, the consequence of which is a further rise in neuroticism. The eventual result can be depression. Researchers from the University of Michigan (Katterman, 1995) found that, in about three quarters of depressed patients, a stressful life event has occurred during the preceding several months.

The experiments of Seligman and his collaborators (Abramson, Seligman, \& Teasdale, 1978; Miller \& Seligman, 1975; Seligman \& Maier, 1967) emphasize the role of learning. They demonstrate that learned helplessness may be a valid model of depression (one of the strongest indicators of neuroticism). The main behavioral symptoms of learned helplessness are a deficit in response initiation and a problem in associating reinforcement with responding. They result from learning that reinforcement and responding are independent from each other. If one has learned from previous experience that there is no connection between reinforcement and responding (i.e., that stressful situations are uncontrollable), this raises the probability that the person will emerge from a stressful situation more vulnerable. On the contrary, if one has learned from previous experience how to associate reinforcement with responding (i.e., how to have active control over the environment), then there is a higher probability that one will be toughened by stressful events (an extensive review on "toughening" and relations to personality is given in Dienstbier, 1989). So, both toughening and learned helplessness are consequences of learning from stressful situations. The bottom line is that, although neuroticism as a basic personality trait strongly depends on genetics, learning through life experience modifies it.

How long does it take to overcome stress? This question can be answered using the time parameter $\tau$ concerning the rate of emotional information processing. This parameter represents the time needed to dissociate the factual information from the affective component and to integrate it in the structured long-term memory from the site of the first storage. We can say that stressful information is accepted when one stops reacting emotionally to the memory of that event. So, at the end of this process, there are two long-term memory traces: one for the facts of the event and one for the emotional reaction. We postulate that the time dependence of emotional information processing is exponential with a characteristic period $\tau$. This raises the question, Is it possible to recognize $\tau$ in human behavior - that is, What is a valid indication of $\tau$ in human behavior and how can it be measured?

Our assumption is that $\tau$ is directly related to another basic trait in the "big five" model of personality-openness. Openness is a broad and general dimension, seen in "vivid fantasy, artistic sensitivity, depth of feeling, behavioral flexibility, intellectual curiosity and unconventional attitudes" (McCrae, 1996). We suppose that the underlying neurophysiological mechanism causing individual differences in openness also plays a crucial role in processing traumatic information. A higher degree of openness means that a person is more cognitively flexible and therefore needs less time (smaller $\tau$ ) to assimilate new information that caused emotional arousal. A low degree of openness characterizes a rigid cognitive system to which we ascribe high $\tau$ (slow processing). A direct prediction of this hypothesized relation is that individuals suffering from posttraumatic stress disorder (PTSD) have low scores on measures of openness.

The next two sections are brief overviews of the HPA axis and memory system functioning, with emphasis on their role in stress response and with references to some of the literature where details can be found. In the third section, we introduce a mathematical model gradually, explaining notions and terms used and citing experimental and clinical results that led us to this model; this Model section is divided into subsections corresponding to the steps of the stress response, which we precisely define. A computer simulation is described in the fourth section; the simulation results are shown in figures. In the fifth section, we discuss the range and limitations of 
the model, with reference to some questions in stress research. In the last section, we point out the essence, purpose, and implications of our model.

\section{THE HPA AXIS}

The HPA axis regularly works at a steady, homeostatic level with a characteristic diurnal rhythm. In the presence of stressors, the HPA axis enhances its work. Stressors can be various - physiological or psychological (differing within these categories, too). Which pathway will be used to activate the stress axis depends on the kind of stressor. When a stressful situation is not a "simple" physiological threat (cold, hunger, pain, hypoxia, etc.), cognitive processing is included. Such stressors are called processive, and they become stressful only by comparison with previous experience (Herman \& Cullinan, 1997; van der Kolk, 1996c). The comparing and attaching of emotional significance to an event is probably done by the amygdala (Herman \& Cullinan, 1997; Roozendaal, Cahill, \& McGaugh, 1996); the limbic system then signals "alarm" to the HPA axis. Limbic stress pathways include the amygdala, prefrontal cortex, and hippocampus. Detailed reviews of HPA axis activation and inhibition pathways are given in Herman and Cullinan (1997), Chrousos and Gold (1992), and Axelrod and Terry (1984). Basically, paraventricular nuclei (PVN) in the hypothalamus generate corticotropin releasing hormone ( $\mathrm{CRH}$ ), which induces the adenocorticotropin (ACTH) production in the pituitary; subsequently, ACTH stimulates the adrenal cortex to produce glucocorticoids, which in turn decrease their own production and the production of both CRH and ACTH. There are many other participants in the stress response mechanism (Axelrod, 1984; Barden, Reul, \& Holsboer, 1995; Chrousos \& Gold, 1992; Dinan, 1996; Feldman \& Weidenfeld, 1996; Folk \& Long, 1988; Herman \& Cullinan, 1997; Jacobs, 1994; Kalicanin \& Lecic-Tosevski, 1994; Paunovic \& Babinski, 1995), but these three form its "backbone." The HPA axis activity intensifies under the influence of a stressing stimulus. We suggest that the stress-aroused concentration change of one (or more) of the HPA axis products helps store the memory about stress. The memory of the stressful event then modifies the threshold for future stressors.

\section{MEMORY SYSTEMS}

Memory and learning mechanisms in humans and animals are subjects of interest for many scientists from various fields: psychologists, psychiatrists, neurologists, computer scientists, and so on. The picture of the information pathways in the brain is far from being completed. Research results indicate the importance of the following memory system structures in processing information: the hippocampal and adjacent perirhinal and parahippocampal cortices (Bremner, Krystal, Southwick, \& Charney, 1995; Squire \& Zola-Morgan, 1991; ZolaMorgan \& Squire, 1990), the prefrontal cortex (Dama- sio, 1994, 1996; Goldman-Rakic, 1996), and the amygdala (Murray \& Mishkin, 1985).

The first phase of learning is rapid (seconds to minutes) compared with the next phase (weeks to months). During the second phase, which is called consolidation (Bremner et al., 1995), memories are gradually being transferred from their initial storage sites to new locations in the neocortex for long-term storage. When consolidation is over, memories become independent of the hippocampal system, as shown in many cases of retrograde hippocampal amnesia (Kim \& Fanselow, 1992; Squire \& Knowlton, 1995; Squire \& Zola-Morgan, 1991; Zola-Morgan \& Squire, 1990). On neuronal level, the mechanism in the hippocampus that accounts for both initial learning and consolidation is long-term potentiation (for a brief overview, see Bear, 1997).

The key difference between rapid and slow learning is in the associations among elements of memories. In the former case, associations are arbitrary, whereas, in the latter, they are meaningful. That is why the long-term "packing" is slow and gradual. McClelland, McNaughton, and O'Reilly (1995) hypothesize that a particular item is learned through a series of presentations interleaved with exposure to other examples from a corresponding domain (stored earlier), and that is why they call this kind of learning interleaved. In such a way, information is classified in categories. Thus, obtained conceptual knowledge forms a firm "structured knowledge system" wherein each new memory gets a proper place determined by similarity to previously acquired concepts.

In the case of emotion-arousing information, it is still unknown to what extent the processing differs from the one described. So far, it is evident that the emotional memorizing process involves the amygdalar complex (Bremner et al., 1995; Cahill, Babinsky, Markowitsch, \& McGaugh, 1995; Calvin, 1990; Damasio, 1994, 1996; Roozendaal, Cahill, \& McGaugh, 1996; Squire \& Zola-Morgan, 1991) and adrenergic systems (Cahill et al., 1995; Cahill, Prins, Weber, \& McGaugh, 1994; Roozendaal, Cahill, \& McGaugh, 1996). The amygdala is the main link between emotions and memories. On one hand, it attaches emotional significance to incoming information and, if needed, alarms the HPA axis (as mentioned in the previous section). On the other hand, storing emotional memories is impossible without it. Thus, it seems logical that the amygdala plays one of the leading roles in forming the stress threshold. But, the apparatus for constituting the stress threshold must be more complex-it must encompass both declarative (facts and events) and nondeclarative (fear conditioning and other emotional responses) memory systems because the categories of objects and situations have to be connected to emotional reactions.

Emotions "catalyze" memorizing via action of the amygdala. The higher the emotional arousal, the deeper the engraving of the memory. On the other hand, too high an arousal inhibits normal hippocampal function in integrating and sorting information (van der Kolk, 1996c; 
van der Kolk \& McFarlane, 1996). The result is a traumatic memory that is isolated (dissociated) from other life experiences and exists in fragments on a more primitive, perceptual level (Bremner et al., 1995; van der Kolk, 1996c), as Janet (1889; see van der Kolk \& van der Hart, 1989) had already observed in 19th century. Traumatic memories do not submit to voluntary control (since they failed to be transformed into declarative form) and are usually triggered in the states of high arousal. Thus, the often mentioned inverted U-shaped function of memories versus emotions applies to hippocampal memory processing (i.e., to memorizing in a declarative form), whereas memorizing in general (in any form) is an all increasing function of emotional arousal. Furthermore, the higher emotional arousal, the less distortion of memory traces.

Damasio's $(1994,1996)$ somatic marker hypothesis about emotional memories deserves mention. Whenever an event arouses emotions, a pattern of neuronal activation (i.e., memory trace) representing the changes in body state emerges in parallel with the factual memory trace; this matching pattern is called the somatic marker. In Damasio's (1994) words, "there is a "combination' rather than a "blending" " of these two patterns that make "a set." The linkages between these two memories are stored in ventromedial prefrontal cortex, which together with amygdala and somatosensory cortices form the network for somatic markers. This network takes part in responses to complex stimuli.

Relating our model to Damasio's, the formation of the stress threshold probably strongly depends on event trace/ somatic marker sets. We can conceive of the stress threshold as a direct function of the ratio of "bad" to "good" (or to the total sum of) markers. The larger the ratio, the more events with negative outcome in the past, and the more likely that the person will anticipate "bad," leading to a reduction of the stress threshold (and inversely). Furthermore, since one of the main somatic changes during stress is cortisol concentration rise, the trace of this change could be the Damasio's somatic marker, and, in our model, it directly influences the change of stress threshold.

\section{THE MODEL}

The aim of this work was to construct a mathematical model of stress reaction based on the assumption of the feedback mechanism between the HPA axis and the memory system using the above-defined individual variables. The following subsections correspond to the steps of the stress response following real order in time.

\section{Stress Excitation}

Although the functioning of the HPA axis is known in greater detail than presented above, for the purposes of this model, it is sufficient to simulate the production and degradation of $\mathrm{CRH}, \mathrm{ACTH}$, and cortisol, a representative of glucocorticoids. Cortisol inl.ibits the production of all three hormones via cortisol receptors in a reaction with saturation of the Michaelis-Menten type (Lauffen- burger \& Linderman, 1996). However, its main effect is to inhibit CRH via glucocorticoid receptors in hypothalamus, hippocampus, and prefrontal cortex (Herman \& Cullinan, 1997; Meaney et al., 1996; Sapolsky, 1996; Seckl et al., 1990).

This mechanism can be described by three differential equations:

$$
\begin{aligned}
& \frac{d C R H}{d t}=k_{C} \cdot\left(1-\eta \cdot \frac{C O R T}{K_{d}+C O R T}\right)-k_{1} \cdot C R H \\
& \frac{d \mathrm{ACTH}}{d t}=k_{1} \cdot C R H-k_{2} \cdot A C T H \\
& \frac{d C O R T}{d t}=k_{2} \cdot A C T H-k_{3} \cdot C O R T,
\end{aligned}
$$

where $C R H, A C T H$, and $C O R T$ represent mean daily concentrations of stress hormones, $k_{C}, k_{1}, k_{2}$, and $k_{3}$ are the reaction rate constants, $K_{d}$ is the dissociation constant of the cortisol-receptor complex, and $\eta$ is a constant whose meaning will be explained below. The first terms on the left signify production, and the second terms (with minus sign) signify decay in the manner of classical chemical kinetics.

As mentioned above, many other factors are involved in the HPA axis mechanism (to name just catecholamines, serotonin, dopamine, gamma-aminobutyric acid), but its main pattern of activity is captured by this simple system. Regular diurnal oscillations of hormone concentrations are ignored, so the concentration changes express only stress dynamics. Numerical values of rate constants $k_{1}=$ $.023, k_{2}=.041$, and $k_{3}=.009$ were calculated from measured half-lives of $\mathrm{CRH}, \mathrm{ACTH}$, and cortisol, respectively (Otero \& Sieburg, 1998).

The rate constant $k_{C}$ is responsible for $\mathrm{CRH}$ production. When there is no source of stress $(S=0)$, the HPA axis functions in its stationary (homeostatic, basal) regime. When a person is in a state of stress $(S>0)$, the activity of the HPA axis is enhanced. This is described by the following equation:

$$
k_{C}=k_{C}^{0} \cdot \exp (S-\sigma) .
$$

In terms of classical chemical kinetics (e.g., Bray \& White, 1957), $k_{C}^{0}$ is an empirical constant, and $\sigma$ corresponds to the activation energy term $E_{\text {act }} / R T$ for the production of CRH ( $R$ is the molar gas constant, and $T$ is absolute temperature). ${ }^{1}$ Energy of activation is minimal energy needed for a reaction to occur (at a given temperature $T$ ), a threshold for a chemical reaction. It determines the reaction rate in equilibrium (stationary) conditions. Knowing that a psychological stressor enhances the level of production of HPA hormones, we mapped the psychological threshold $\sigma$ to the onset of activating the HPA axis (i.e., to the activation energy for the production of CRH, the first reaction in the HPA cascade).

The quantity $S$ is an individual measure of the action of an external stressor $\left(S_{\mathrm{e}}\right)$, which is experienced only in comparison with the stress threshold: $S=S_{\mathrm{e}}-\sigma\left(S_{\mathrm{e}}>\sigma\right)$. 
The larger the stressor, the higher the level of HPA axis functioning. In this sense, $S$ corresponds to the energy brought to the system.

There is no instrument for direct and objective measuring of external stressors, only scales for estimating stressor intensity (standard scale is multiaxial DSM-III-R system). The scales are formed on statistical basis: Personal estimations are averaged over a population. The evaluations of stressor strength are made indirectly, through the intensity of emotional reactions the stressors provoke. In other words, $S_{\mathrm{e}}$ is the intensity of a stressor on an arbitrary scale calibrated by average intensities of emotional responses in a population. Threshold $\sigma$ is the intensity of a minimal stressor arousing emotional response in an individual. Thus, the "units of measure" for $\sigma, S$ and $S_{\mathrm{e}}$ are the same. Obviously, $S_{\mathrm{e}}$ can be less than a person's $\sigma$, but in this case it does not have a stressful effect on the individual.

Before an external stressor starts to act, the HPA axis functions in its homeostatic (stationary) regime. We assume that the basic production of CRH depends on $\sigma$ that is, that the basic level of HPA axis is correlated with one's general vulnerability (depending on genetic factors and modified by previous experience). We further assume that the HPA axis works at a threshold level, ready to switch to "alarm regime" at the appearance of an external stressor. This readiness to react makes the stress system a highly adaptive (complex) system.

\section{Regulation of the Immediate Response}

Glucocorticoids have one more important function: to regulate the HPA axis intensity. Some investigators even think that this is their primary role (Piazza \& Le Moal, 1997). This is accomplished by the negative feedback loops that act on all three levels of the HPA axis via corticoid $^{2}$ receptors, CRs (Meaney et al., 1996; Roozendaal, Bohus, \& McGaugh, 1996; Sapolsky, 1996; Seckl et al., 1990; Yehuda, Teicher, Trestman, Levengood, \& Siever, 1996). The most important regulatory loop includes formation of the glucocorticoid-receptor complex (GRC), which stops the hypersecretion of $\mathrm{CRH}$. This regulating function is represented by the term $\eta \cdot \operatorname{CORT} /\left(K_{d}+\right.$ $C O R T$ ) in Equation 1. When CORT $\gg K_{d}$, this function approaches saturation (i.e., most receptors are occupied, and further secretion of the glucocorticoids does not influence the $\mathrm{CRH}$ production). When $\mathrm{GRC} \rightarrow \mathrm{CR}_{\mathrm{tot}}$ (total number of CRs, taken to be constant during the regulation of the immediate response) - that is, when the inhibition (suppression) is maximal- $k_{c}$ is decreased for a maximal fraction $\eta$. Thus, $\eta$ is a constant between 0 and 1 , directly depending on $\mathrm{CR}_{\text {tot }}$. It is obvious that a large number of receptors provides better regulation of the stress system because saturation will be reached for a larger cortisol concentration. The limit of HPA "elasticity" is thus determined by the number of CRs acting on hypothalamic CRH-producing cells. In other words, a person will be able to compensate for a larger stress if his/her $\mathrm{CR}_{\text {tot }}$ is larger. The influence of early postnatal environ- ment on receptor development is crucial for CR production (Meaney et al., 1996). The regulation of HPA axis can be disturbed under conditions of chronic stress.

\section{Sensitization}

The threshold $\sigma$ depends on previous experiencemainly, previous stresses. Each stressful experience is marked by a change in cortisol concentration, which helps it to be memorized, and, subsequently, each such experience modifies $\sigma$. Since the steps of this process are not known, we condense them in one phenomenological equation:

$$
\Delta \sigma=-k_{4} \cdot \triangle C O R T,
$$

where $\Delta \sigma$ represents the change of $\sigma$ after the action of the external stressor, and $k_{4}$ is the rate constant of this complex process (the number of corticoid receptors, mainly in the limbic system, must play a role in $k_{4}$ ). This equation closes the loop in the feedback mechanism of the two systems. The higher the stress, the larger the stressinduced cortisol concentration change ( $\triangle C O R T)$, and the lower the stress threshold for future stimuli (not necessarily of the same kind). The new threshold value will be $\sigma=\sigma_{0}+\Delta \sigma\left(\sigma_{0}\right.$ is the initial value of stress threshold $)$, keeping in mind that $\Delta \sigma<0$. In other words, the inverse dependence given by Equation 5 describes general sensitization by a stressful event. Most people show nonspecific hyperarousal in the immediate aftermath of a traumatic event (Bremner et al., 1995; Kalicanin \& LecicTosevski, 1994; Shalev, 1996; Solomon, Laror, \& McFarlane, 1996; van der Kolk, 1996a; 1996b; van der Kolk \& McFarlane, 1996), meaning that this change is temporary. Solomon et al. (1996) emphasize that "the acute stress reaction constellation is almost universal," with undifferentiated fear as its primary determinant. Actually, there is a latent period of several hours to several days (Kalicanin \& Lecic-Tosevski, 1994; Shalev, 1996) between the event and the appearance of sensitization (see Discussion section below).

The link between cortisol and vulnerability is also supported by clinical data on hypercortisolemia, which is found in the vast majority of depressive and PTSD patients $^{3}$ (Anisman \& LaPierre, 1982; Barden et al., 1995; Goenjian et al., 1996; Sapolsky, 1996; Seckl et al., 1990; Yehuda \& Sapolsky, 1997; Yehuda et al., 1996). Both of these types of patients are known to be more vulnerable than average (Brett, 1996; Halbreich et al., 1989; Orr, 1994; van der Kolk \& McFarlane, 1996; van der Kolk, van der Hart, \& Marmar, 1996; van der Kolk, Weisaeth, $\&$ van der Hart, 1996). In a study on hospitalized children, Knight et al. (1979) found that effective coping was related to lower cortisol levels. Ursin, Baade, and Levine (1978) noted correspondence between high cortisol responses and defensiveness with paratrooper trainees.

So, Equation 5 reflects the assumption that some product(s) of the HPA axis helps a stressful event be memorized. Cortisol seems to be the best candidate for the role of "messenger" from HPA axis to the memory system for 
several reasons: (1) glucocorticoids are known to enhance memory storage ${ }^{4}$ (Korneyev, 1997; Roozendaal, Cahill, \& McGaugh, 1996); (2) a correlation between its secretion and vulnerability has been observed, as mentioned above; and (3) cortisol receptors are present in hippocampus and neocortex.

We want to emphasize again that cortisol was chosen only as a measure of the HPA axis activity; it is not necessarily the direct memory catalyst itself. It is a convenient marker because it is easy to measure due to its abundance and relatively long life $\left(\tau_{1 / 2} \cong 90 \mathrm{~min}\right)$. Even if it is only a link in the chain, it may serve as an indicator of some other direct or indirect product of HPA axis, provided that their concentrations are in proportion.

\section{Recovery}

Besides differences in $\sigma$, individuals differ in the time needed to assimilate stressful information. The larger the stress, the longer it takes to be assimilated. We assume that as long as the process of adopting the information lasts, a person is in the state of stress. When the external stressor is eliminated, the memory of this event acts as an internal source of stress. From the site of the first storage, the stressful information is gradually processed and stored in the structured memory system-that is, it becomes consolidated. During this process, the "emotional component" of the information detaches from the "factual component," and the stress state attenuates. All this happens with individual rate $1 / \tau$ ( $\tau$ is a personal measure of time needed for the stress experience to be digested, and it depends on personality traits-mainly, openness). According to Loehlin (1992), "openness variables showed the largest estimates for additive genetic variance ... of any of the Big Five" (pp. 66), so we suppose that genetic factors are decisive in $\tau$.

Taking stress as a disturbance in a system, we simulate a decline in stress as the result of system's tendency to return to its stationary state. Relaxation processes usually follow the exponential law:

$$
S=\left(S_{\mathrm{e}}-\sigma_{0}\right) \cdot \exp (-\mathrm{t} / \tau)
$$

In the course of the same process (so the time dependence is the same), stress threshold increases again. But, the final outcome-whether a person comes out of a stressful situation as sensitized or toughened-depends on the success of coping:

$$
\sigma=\sigma_{0}+\Delta \sigma \cdot \exp (-t / \tau)+k_{5} \cdot \Delta \sigma \cdot \ln \left(N_{+} / N_{-}\right),
$$

where $N_{+}$is the number of positively estimated reactions in one's life experience, $N_{-}$is the number of negatively estimated reactions (in the context explained in the introduction), and $k_{5}$ is a proportionality constant. The second term of Equation $7[\Delta \sigma \cdot \exp (-t / \tau)]$ shows the decrease of the temporary change of $\sigma$. Higher $\tau$ indicates slower recovery from the transient sensitization. The last term is responsible for long-lasting effects of stress: if $N_{+} / N_{-}>1$ (more successful than failed copings in past, as estimated by the person in question), then $\ln N_{+} / N_{-}>$
$0, \sigma$ increases (i.e., a person is toughened by stressful experience). $N_{+} / N_{-}<1\left(\ln N_{+} / N_{-}<0\right)$ means a decrease in $\sigma$ (i.e., long-term increase in vulnerability). We name the term $\ln N_{+} / N_{-}$the coping factor.

Processing of the stressful memory influences the HPA axis through the rate constant of $\mathrm{CRH}$ production (Equation 4). The difference $(S-\sigma)$ in Equation 4 decreases because of both descending $S$ and recovery of $\sigma$. The recovery of $\sigma$ is relevant only for subsequent stressors. In Equation 6, it stays at the value $\sigma_{0}$, because $\left(S_{\mathrm{e}}-\sigma_{0}\right)$ represents the initial stressor intensity: $S_{\mathrm{e}}$ found the system at $\sigma_{0}$, and it cannot be changed retroactively. $S$ decreases only due to the term $\exp (-t / \tau)$.

To summarize, Equations 1-3 represent the stress dynamics of the HPA axis, Equations 6 and 7 represent processes in the memory system, and Equations 4 and 5 reflect processes that link these two systems: Equation 4

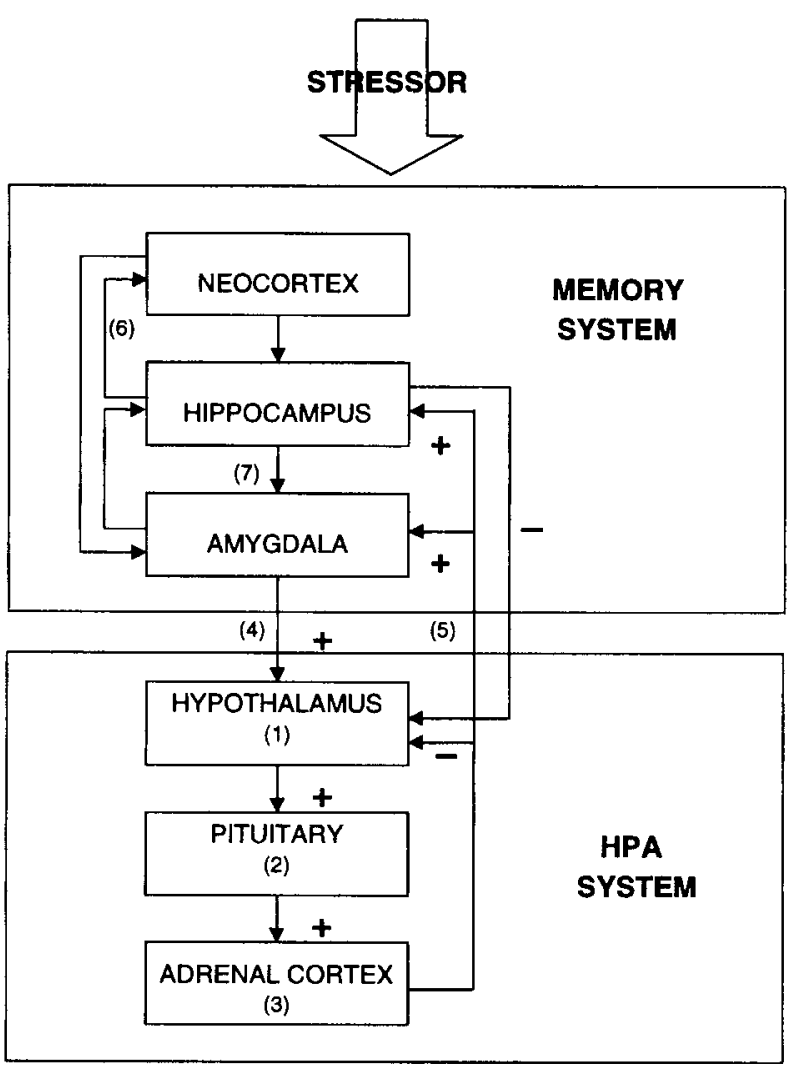

Figure 1. A schematic representation of the model: parts of the memory system, the HPA axis, and some of their links. The numbers in parentheses designate equations (given in text) and are placed at supposed sites of the described processes: Equations 1, 2 , and 3 concern the kinetics of $C R H, A C T H$, and cortisol, respectively, so they are placed at the sites of their production. Equation 4 shows the activation of HPA axis by the memory system mediated by amygdala, and Equation 5 describes the influence of HPA stress response on the processes in the memory system. Equation 6 is mainly related to the consolidation of memories. We suppose that the process given by Equation 7 mainly takes place at hippocampus, and amygdala transfers its result (the new value of stress threshold) to hypothalamus at the appearance of a new stressor. 
shows the influence of memory on the HPA axis, and Equation 5 describes the process of opposite direction (see Figure 1).

\section{SIMULATION}

Resuming the above-cited literature, we can say that the stress reaction is roughly a three-stage process operating on three time scales. The sequence of steps is the following: (1) immediate (within minutes) response of the HPA axis, the secretion of stress hormones and self-regulation by glucocorticoids; (2) increase of psychological and physiological sensitivity (decrease of the stress threshold) when the action of the external stressor is over, starting several hours to several days after the event; (3) decrease of internal stressor intensity and recovery of the stress threshold, lasting weeks to months after the event.

Stage 1: In the simulation, we first let the system (Equations $1-3)$ stabilize in a stationary state $(S=0)$ given the rate constants and $\sigma_{0}$. Then, we "stress" it with $S_{\mathrm{e}}>\sigma_{0}$ for a short time period $\left(S=S_{\mathrm{e}}-\sigma_{0}\right)$. The levels of $C R H$, $A C T H$, and $C O R T$ jump as an immediate response of the HPA axis system. The inhibitory action of cortisol occurs (the inhibition term in Equation 1) but is overridden to a great extent by the action of $S$.

Stage 2: The external stressor is removed, and sensitization begins. $\sigma$ drops proportionally to the increase in the cortisol concentration (Equation 5 added).
Stage 3: Recuperation begins $\left(t=0, S=S_{\mathrm{e}-} \sigma_{0}\right)$-that is, the memory of trauma (internal stressor) is gradually being stored separately from the emotional component. $S$ decreases and $\sigma$ increases again with the rate $1 / \tau$ to a new value, as a result of the stressful experience (Equations 6 and 7 added).

Finally, CORT descends $\left(k_{c}\right.$ decreases as $S-\sigma$ decreases).

When the system reestablishes a steady state, on a new cortisol (i.e., threshold) level, the whole process repeats three more times, simulating three stressful experiences.

The results of the simulations are shown in Figures 2, 3 , and 4 .

\section{Discussion}

There are two recent mathematical models on diurnal functioning of the HPA axis (Gonzalez-Heydrich, 1994; Otero \& Sieburg, 1998). On the other hand, there are models on fear conditioning (Armony, Servan-Schreiber, Cohen, \& LeDoux, 1995; Armory, Servan-Schreiber, Romanski, Cohen, \& LeDoux, 1997). Gonzalez-Heydrich's model has a similar mathematical approach to ours, whereas the others are neural network models. However, none of them concerns the feedback of HPA axis and memory system. Also, the question of individual differences is not addressed.

Our model offers a possible (simplified) correlation between the HPA axis and the memory system. The idea

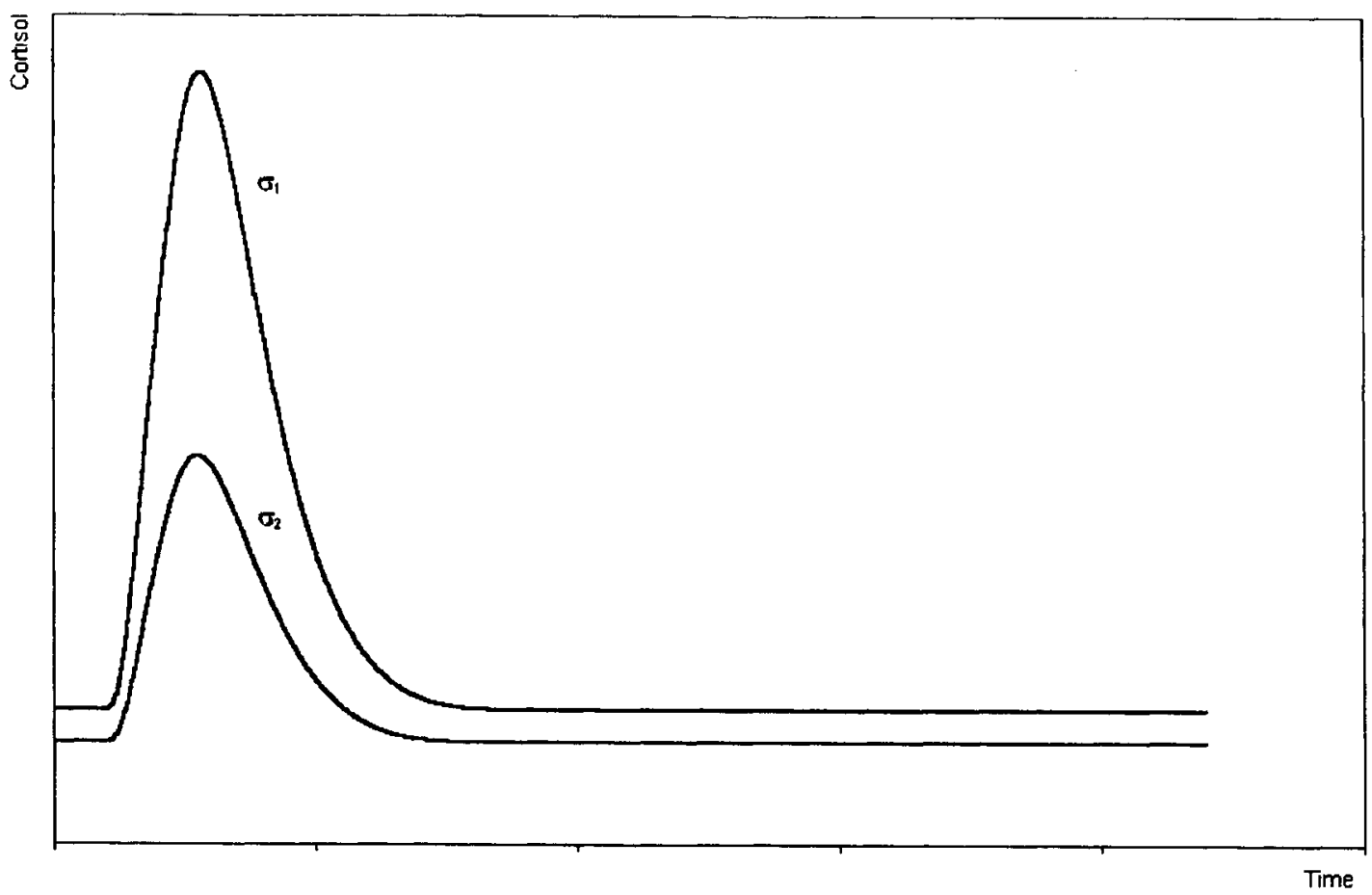

Figure 2. Rise and recovery of cortisol concentration during stress in two hypothetical individuals differing only in stress threshold $\left(\sigma_{1}<\sigma_{2}\right)$ : The intensity of the external stressor is the same, but the individual with the lower $\sigma$ experiences it as more stressful (higher cortisol peak). 


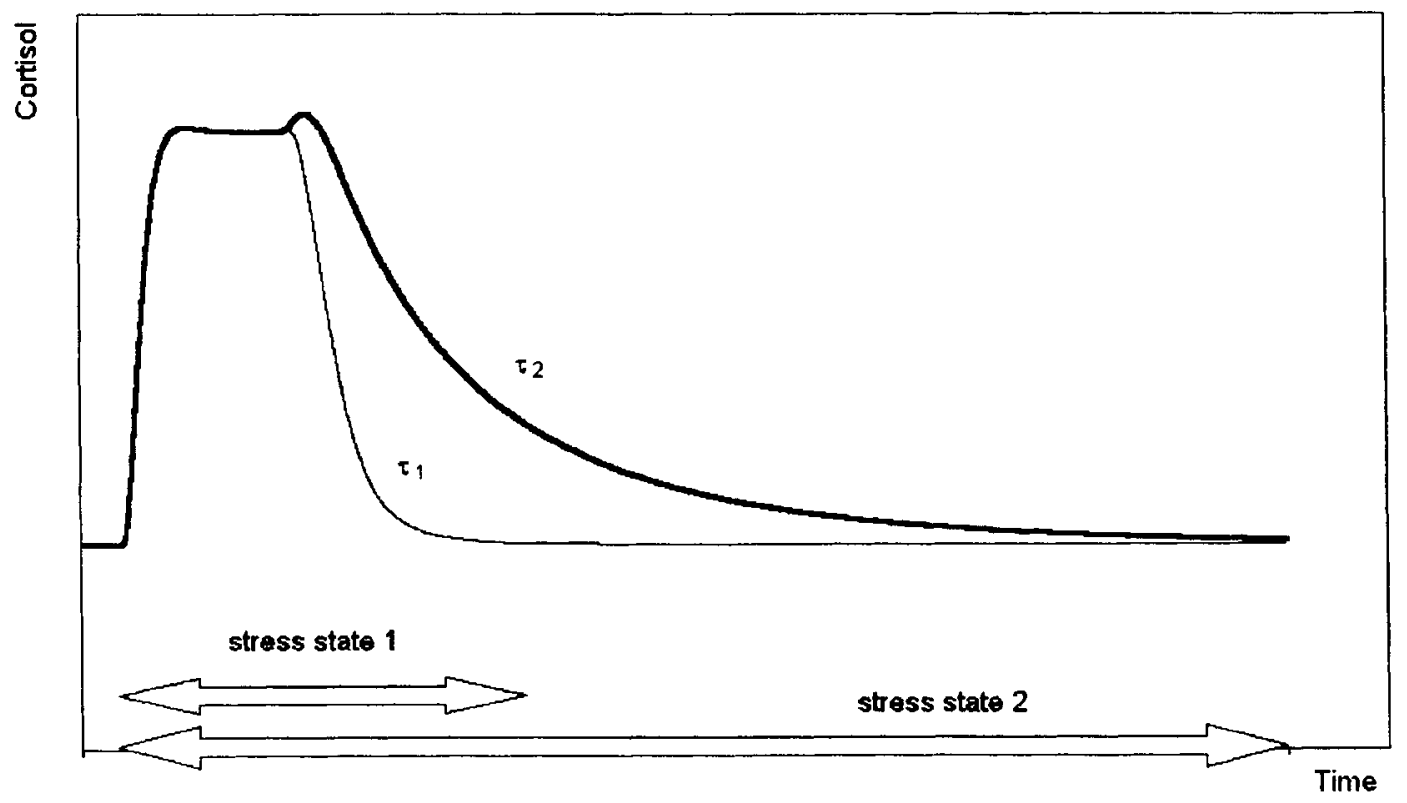

Figure 3. Rise and recovery of cortisol concentration during stress in two hypothetical individuals differing only in characteristic recovery time $\left(\tau_{1}<\tau_{2}\right)$ : The intensity of the external stressor is the same, but the individual with higher $\tau\left(\tau_{2}\right)$ spends more time in the state of stress. Although the model accounts for short actions of external stressors, the acute phase of stress (external stressor present) is extended to show the phases of stress: If the action of external stressor lasts, a temporary "alarm" steady state is established (the plateau). The small peak after the plateau is cortisol rise due to sensitization-it occurs in the individual with $\tau_{2}$. If $\tau$ is very short $\left(\tau_{1}\right)$, the recovery process interferes with sensitization not letting it develop fully. The influence of $\tau$ on sensitization was not presupposed, it is purely a result of the simulation. This means that more "open" individuals not only will recover faster but are even less sensitized than the rigid ones.

of feedback between these two systems can be found in the literature on stress and is formulated in different ways by different authors. An explicit and clear formulation is given by Roozendaal, Cahill, and McGaugh (1996):

Events that are sufficiently arousing to elicit emotional reactions and behavioral responses are also, of course, likely to be worth remembering. And memory of the experiences can elicit appropriate reactions to subsequent exposure of the same or similar experience. The thesis of this chapter is that the same hormonal components of the stress response that prepare the animal to respond immediately to an emotionally arousing experience are also involved in regulating the storage of memory of the experience. (p. 39)

Our model describes individual stress reaction to discrete and relatively short traumatic incidents based on this attitude. We can say that $\sigma$ is the meeting point of the two systems, thus directly connecting physiological and psychological processes: On one hand, it is the result of a complex process of learning from stressful situations that includes the influence of enhanced stress hormone concentrations; on the other hand, it determines the level of HPA axis activity. Although the precise steps of forming the stress threshold are still unknown, the correlation between $\sigma$ and cortisol concentration is observed and is emphasized here.
What exactly happens during an acute stressful situation (impact phase of stress) is not well known because the only available sources of data are personal reports. Also, frequently repeated and long-lasting stressors can have cumulative effects that often distort the response mechanism (McFarlane \& de Girolamo, 1996), a case this model does not address.

There is an ongoing debate about the differences between nontraumatic and traumatic stress (Shalev, 1996). Lately, differences have been sought in subjective factors (i.e., in individual reactions) instead of objective factors (the type and intensity of stressor). This is the assumption on which our model is based. In particular, we assume that a person experiences the intensity of a stressor only to the extent that it exceeds his/her threshold. As mentioned in the Memory Systems section, one of the main differences between stress and traumatic stress is in processing stressful information. There is no integration of sensory elements into a personal narrative in the latter case, leading to dissociation of traumatic memories (van der Kolk, 1996c; van der Kolk \& McFarlane, 1996). It seems that too intense emotions (hyperorousal of amygdala) inhibit some hippocampal memory functions (van der Kolk, 1996c). There might be another individual threshold for traumatic stress $\left(\sigma_{t}\right)$, an intensity of stress that overpowers one's coping mechanisms. In 

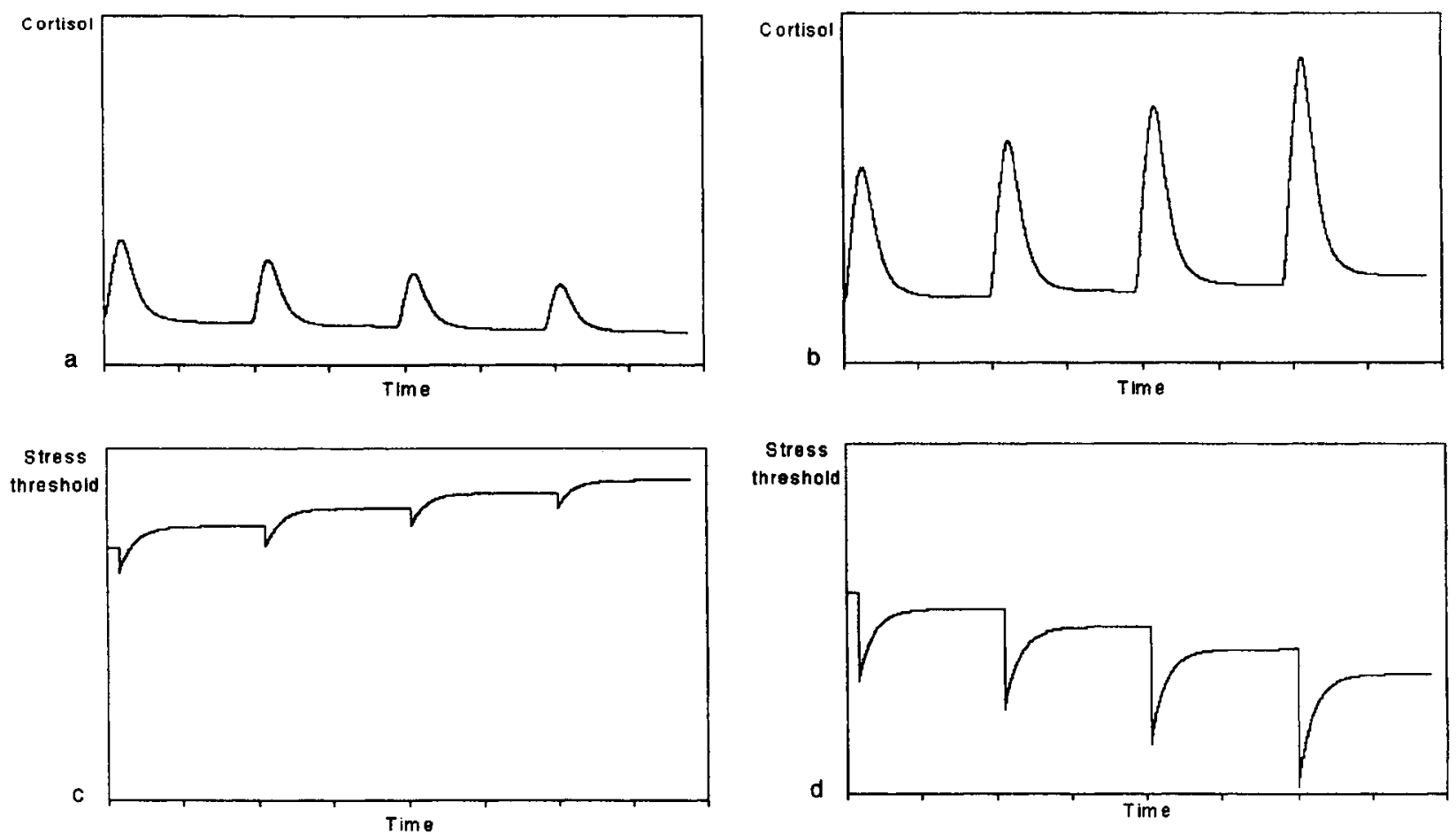

Figure 4. Two hypothetical individuals having the same $\tau$, but differing in $\sigma_{0}$ and coping factor, exposed to four stressors of identical intensity in identical time intervals (measured in months/years). Panels a and b show their cortisol levels as functions of time, and Panels $c$ and $d$ show the respective changes of their stress thresholds during the same time period. $\sigma$ (a) $>\sigma$ (b). Left-side graphs (Panels a and c) represent an individual with higher $\sigma$ and with a positive coping factor. This individual's starting reaction is not only smaller but it diminishes in repeated stressful situations due to the positive coping factor. The basal cortisol level (between the peaks) in this case is also decreasing. This individual is toughened by stresses. The case on the right side (Panels b and d) is the contrary: a person high on neuroticism (low $\sigma$ ) undergoes a dangerous feedback loop—he/she evaluates his/her coping as unsuccessful (negative coping factor), which causes lowering in $\sigma$ (d) and consequently a higher response to repeated stressors followed by increase of basal cortisol level (b). Sharp declines of os represent temporary sensitizations, after which thresholds recover to new values.

terms of this model, it means that Equations 6 and 7 are valid for the range of $S_{\mathrm{e}}: \sigma<S_{\mathrm{e}}<\sigma_{\mathrm{t}}$, if $\sigma_{\mathrm{t}}$ exists. The inhibition of hippocampal functions during intense HPA activity may also account for the supposed lag time of the onset of sensitization, on the basis of the clinical evidence referred to earlier (The Model section, Sensitization subsection). This phenomenon has a biological justification in that a person's survival is endangered if he or she becomes more and more vulnerable during the impact phase of stress.

Recovery processes are described (in Equations 6 and 7) by an exponential function of time, meaning that cortisol is also monotonously descending (i.e., its concentration is averaged during this period). A more precise description would be a function with irregular attenuated oscillations. The amplitudes of this function would correspond to intrusions of stressful memories. With progression of recovery, the amplitudes should diminish, and the intervals between them should grow longer. However, the essence of the process is preserved in this approximation.

What happens if a new $S_{\mathrm{e}}$ occurs during the recovery period? In spite of being hypersensitive after a traumatic event, depressive and PTSD patients during, respectively, depressive and intrusive episodes are insensitive to external stressors that would otherwise provoke a reaction. Chrousos and Gold (1992) claim that painful memories make the stress system refractory to external stressors. This implies that stressors are not additive, but the system reacts to the strongest of them (at least when it is extremely intense). As mentioned in the HPA Axis section, the amygdala is hypothesized to be the site at which the incoming external stressor is compared with the contents of memory to determine whether or not its intensity is above the threshold. The amygdala might as well be the comparator of external and current internal stressor, if there is one, to determine which one is stronger.

Note that there are two equations (Equations 5 and 7) expressing the changes of $\sigma$, meaning that we assume two different processes responsible for these changes, including different locations in the brain. It is likely that information that is built in $\sigma$ comes from both the sites of initial storage (the hippocampus) and the structured memory "drawers" (the neocortex). If there is a recent stressful memory, it probably has a prevalence over the old memories in forming the $\sigma$. The rapid change of $\sigma$ due 
to the new stressor (sensitization) is a part of the rapid learning process, represented by Equation 5 . We modeled it as an instantaneous process relative to subsequent recovery, which is a part of interleaved learning, represented by Equations 6 and 7.

\section{CONCLUSIONS}

This work connects psychological and biological aspects of stress reaction. It integrates a wide range of results from diverse research fields (physiology, psychology, psychiatry, etc.) into a consistent feedback mechanism between the HPA axis and the memory system in stress reaction.

Two variables- $\sigma$ and $\tau$-are extracted as the main carriers of individual differences, representing two basic personality traits - neuroticism and openness-which relate memory system to stress axis.

This work is also a trial to define precisely the notions related to stress and their interrelations so they could be operational and translated into mathematical variables and functions. Using thus defined quantities, the complex mechanism of stress reaction is described by seven equations.

The model has two important implications (predictions): (1) that there is a direct correlation between the vulnerability and the basic cortisol level in nonstressed individuals (via quantity $\sigma$ ); and (2) that individuals who have prolonged acute stress reaction (high $\tau$ ) and suffer of intrusive memories, particularly PTSD sufferers, have low scores on openness.

In our future research, we intend to examine the first implication experimentally, parallelly testing a normal (nontraumatized) population, by endocrine analyses and personality questionnaires. We are in the process of testing the second implication on a war-stressed population. Our plans also include examining the role of corticoid receptors as the third important determinant of individual differences.

In spite of having many gaps to fill in, we hope that this model offers a useful framework for future investigations of the mechanism operating on the HPA axis and the memory system during stress. This model should be conceived only as a starting point in theoretical/mathematical approach to this complex problem, and we hope to develop it in interactions with empirical/experimental research.

\section{REFERENCES}

Abramson, L. Y., Seligman, M. E. P.. \& Teasdale, J. D. (1978), Learned helplessness in humans: Critique and reformulation. Journal of Abnormal Psychology, 87, 49-74.

Anisman, H., \& LaPiERrE, Y. (1982). Neurochemical aspects of stress and depression: Formulations and caveats. In R. W. Neufeld (Ed.), Psychological stress and psychopathology (pp. 179-217). New York: McGraw-Hill.

Armony, J. L., Servan-Schreiber, D., Coh. N, J. D., \& LeDoux, J. E. (1995). An anatomically constrained neural network model of fear conditioning. Behavioral Neuroscience, 109, 246-257.
Armony, J. L., Servan-Schreiber, D., Romanski, L. M., Cohen, J. D., \& LeDouX, J. E. (1997). Stimulus generalization of fear responses: Effects of auditory cortex lesions in a computational model and in rats. Cerebral Cortex, 7, 157-165.

AXELROD, J. (1984). The relationship between the stress hormones, catecholamines, ACTH and glucocorticoids. In E. Usdin, R. Kvetnansky, \& J. Axelrod (Eds.), Stress--The role of catecholamines and other neurotransmitters (Vol. 1, pp. 3-13). New York: Gordon \& Breach.

AXELroD, J., \& TerRY, D. R. (1984). Stress hormones: Their interaction and regulation. Science, 224, 452-459.

Barden, N., Reul, J. M. H. M., \& Holsboer, F. (1995). Do antidepressants stabilize mood through actions on the HPA system? Trends in Neurosciences, 18, 6-10.

BEAR, M. F. (1997). How do memories leave their mark? Nature, 385 , $481-482$.

Bray, H. G., \& WhITE, K. (1957). Kinetics and thermodynamics in biochemistry. London: J. \& A. Churchill.

Bremner, J. D., Krystal, J. H., Southwick, S. M., \& Charney, D. S (1995). Functional neuroanatomical correlates of the effects of stress on memory. Journal of Traumatic Stress, 8, 527-553.

BRETT, E. A. (1996). The classification of posttraumatic stress disorder. In B. A. van der Kolk, A. C. McFarlane, \& L. Weisaeth (Eds.), Traumatic stress: The effects of overwhelming experience on mind, body. and society (pp. 117-128). New York: Guilford.

Cahill, L., Babinsky, R., Markowitsch, H. J., \& McGaugh, J. L. (1995). The amygdala and the emotional memory. Nature, 377 , 295-296.

Cahill, L., Prins, B., Weber, M., \& McGaugh, J. L. (1994), $\beta$-adrenergic activation and memory for emotional events. Nature, 371, 702-704.

Calvin, W. H. (1990). The cerebral symphony. New York: Bantam.

CATtELl, R. (1970). The scientific analysis of personality. Harmondsworth, U.K.: Penguin.

Chrousos, G. P., \& GolD, P. W. (1992). The concepts of stress and stress system disorders. Journal of the American Medical Association, 267. 1244-1252.

Cloninger, R. (1987). A systematic method for clinical description and classification of personality variants. Archives of General Psychiatry, 44, 573-587.

COSTA, P. T., \& MCCRAE, R. R. (1992). The revised NEO Personality Inventory (NEO-PI-R) and NEO Five-Factor (NEO-FFI) professional manual. Odessa, FL: Psychological Assessment Resources.

Cox, T., \& Ferguson, E. (1991). Individual differences, stress and coping. In C. L. Cooper \& R. Payne (Eds.) Personality and stress: Individual differences in the stress process. Chichester, U.K.: Wiley.

DAMASio, A. R. (1994). Descartes 'error: Emotion, reason, and the human brain. New York: Grosset/Putnam.

Damasio, A. R. (1996). The somatic marker hypothesis and the possible function of the prefrontal cortex. Philosophical Transactions of the Royal Society of London: Series B, 351, 1413-1420.

DienstBier, R. A. (1989). Arousal and physiological toughness: Implications for mental and physical health. Psvchological Review, 96, 84-100.

Dinan, T. G. (1996). Serotonin and the regulation of hypothalamicpituitary-adrenal axis function. Life Sciences, 58, 1683-1694.

Eysenck, H. J., \& Eysenck, S. B. G. (1969). Personality structure and measurement. London: Routledge \& Kegan.

FELDMAN, S.. \& WEIDENFELD, J. (1996). Involvement of amygdalar alpha adrenoceptors in hypothalamo-pituitary-adrenocortical responses. Neuroendocrinology, 7, 3055-3057.

FOLK, G. E., \& LoNG, J. P. (1988). Serotonin as a neurotransmitter; A review. Comparative Biochemistry \& Physiology, 91C, 251-257.

Goenjian, A. K., Yehuda, R., Pynoos. R. S., Steinberg, A. M.. Tashilan, M., Yang, R. K., Najarian, L. M., \& Fairbanks, L. A. (1996). Basal cortisol, dexamethasone suppression of cortisol, and MHPG in adolescents after the 1988 earthquake in Armenia. American Journal of Psychiatry, 153, 929-934.

Goldman-RAKIC, P. S. (1996). The prefrontal landscape: Implications of functional architecture for understanding human mentation and the central executive. Philosophical Transactions of the Roval Society of London: Series B, 351, 1445-1453. 
Gonzalez-Heydrich, J. (1994). A computer simulation of the hypothalamic-pituitary-adrenal axis. http://www.chip.org/chip/projects/ hpa/paper-hpa.html

Guilford, J. P. (1959), Personality. New York: McGraw-Hill.

Halbreich, U., Olympia, J., Carson S., Glogowski, J., Yeh, C., AxELROD, S., \& DESU, M. M. (1989). Hypothalamo-pituitary-adrenal activity in endogenously depressed post-traumatic stress disorder patients. Psychoneuroendocrinology, 14, 365-370.

Herman, J. P., \& Cullinan, W. E. (1997). Neurocircuitry of stress: Central control of the hypothalamo-pituitary-adrenocortical axis. Trends in Neurosciences, 20, 78-84.

JACOBS, B. L. (1994). Serotonin, motor activity and depression-related disorders. American Scientist, 82, 456-463.

JANET, P. (1889). L'automatisme psychologique: Essai de psychologie expérimentale sur les formes inférieures de l'activité humaine. Paris: Felix Alcan.

JANET, P. (1909). Problèmes psychologiques de l'émotion. Revue Neurologique, 17, 1551-1687.

Kalicanin, P., \& Lecic-Tosevski, D. (1994). The book about stress. Belgrade: Medical Book.

Katterman, L. (1995). When the stress axis goes tilt. University of Michigan Research News, 46, 7.

KIM, J. J., \& FANSELOW, M. S. (1992). Modality-specific retrograde amnesia for fear. Science, 256, 675-677.

Knight, R. B., Atkins, A.. Eagle. C. J., Evans, N., Finkelstein, J. W., Fuxushima, D., Katz, J., \& Weiner, H. (1979). Psychological stress, ego defenses, and cortisol production in children hospitalized for elective surgery. Psychosomatic Medicine, 41, 40-49.

KORNEYEV, A. Y. (1997). The role of the hypothalamic-pituitaryadrenocortical axis in memory-related effects of anxiolytics. Neurobiology of Learning \& Memory, 67, 1-13.

LaufFenburger, D. A., \& Linderman, J. J. (1996). Receptors: Models for binding, trafficking, and signalling (pp. 216-221). New York Oxford University Press.

LoEHLIN, J. C. (1992). Genes and environment in personality development. Newbury Park, CA: Sage.

McClelland, J. L.. McNaughton, B. L.. \& O'Reilly, R. C. (1995) Why there are complementary learning systems in the hippocampus and neocortex: Insights from the successes and failures of connectionist models of learning and memory. Psychological Review, 102, 419-457.

MCCRAE, R. R. (1996). Social consequences of experiential openness. Psvchological Bulletin, 120, 323-337.

MCFari.ANE, A. C., \& DE Girolamo, G. (1996). The nature of traumatic stressors and the epidemiology of posttraumatic reactions. In B. A van der Kolk, A. C. McFarlane, \& L. Weisaeth (Eds.), Traumatic stress: The effects of overwhelming experience on mind, body, and society (pp. 129-154). New York: Guilford.

McFarlane, A. C.. \& Yehuda, R. (1996). Resilience, vulnerability and the course of posttraumatic reactions. In B. A. van der Kolk, A. C. McFarlane, \& L. Weisaeth (Eds.), Traumatic stress: The effects of overwhelming experience on mind, bodv, and societv (pp. 155-181). New York: Guilford.

Meaney, M. J., Diorio, J., Francis, D., Widdowson, J., LaPlante, P., Caldi, C., Sharma, S., Seckl, J. R., \& Plotsky, P. M. (1996). Early environmental regulation of forebrain glucocorticoid receptor gene expression: Implications for adrenocortical responses to stress. Developmental Neuroscience, 18, 49-72.

Miller. W. R., \& Seligman, M. E. P. (1975). Depression and learned helplessness in man. Journal of Abnormal Psychology, 84, 228-238.

Momirovic, K., Wolf, B., \& Dzamonja, Z. (1992). KON 6: Battery of conative tests. Belgrade: Psychological Association of Serbia.

MurRay, E. A., \& Mishkin, M. (1985). Amygdalectomy impairs crossmodal association in monkeys. Science, 228, 604-605.

ORR, S. P. (1994). An overview of psycho-physiological studies of PTSD. PTSD Research Quarterly, 5(1), 1-7.

Otero, J. M., \& Sieburg, H. B. (1998). Emergent patterns of HPA hormone pulsatility and diurnal variability in silico. http://www. netsci.org/Science/Bioinform/feature02.html; http://www.netsci.org/ Science/ Bioinform/feature 02b.html; http://www.netsci.org/Science/ Bioinform/feature $02 \mathrm{c} . \mathrm{html}$

Paunovic, V. R., \& Babinski, T. (1995). Biological psvchiatry 1: The molecular basis of mental processes. Belgrade: Faculty of Medicine, University of Belgrade.

Piazza, P. V., \& LE Moal, M. (1997). Glucocorticoids as a biological substrate of reward: Physiological and pathophysiological implications. Brain Research Reviews, 25, 359-372.

RoozendaAl, B., Bohus, B., \& McGaugh, J. L. (1996). Dose-dependent suppression of adrenocortical activity with metyrapone: Effects on emotion and memory. Psychoneuroendocrinology, 21, 681-693.

Roozendal, B., Cahill, L., \& MCGaugh, J. L. (1996). Interaction of emotionally activated neuromodulatory systems in regulating memory storage. In K. Ishikawa, J. L. McGaugh, \& H. Sakata (Eds.), Brain processes and memory (pp. 39-54). Amsterdam: Elsevier.

SAPOLSKY, R. M. (1996). Why stress is bad for your brain. Science, 273 749-750.

Secki., J. R., Campbell, J. C., Edwards, C. R. W., Christie, J. E., Whalley, L. J., Goodwin, G. M., \& FinK, G. (1990). Diurnal variation of plasma corticosterone in depression. Psychoneuroendocrinology, 15, 485-488.

Seligman, M. E. P., \& Maier, S. F. (1967). Failure to escape traumatic shock. Journal of Experimental Psychology, 74, 1-9.

Shalev, A. Y. (1996). Stress versus traumatic stress: From acute homeostatic reactions to chronic psychopathology. In B. A. van der Kolk, A. C. McFarlane, \& L. Weisaeth (Eds.), Traumatic stress: The effects of overwhelming experience on mind, body, and society (pp. 77-101) New York: Guilford.

Solomon Z., Laror, N., \& McFarlane A. C. (1996). Acute posttraumatic reactions in soldiers and civilians. In B. A. van der Kolk, A. C. McFarlane, \& L. Weisaeth (Eds.), Traumatic stress: The effects of overwhelming experience on mind, body, and societv (pp. 102-114). New York: Guilford.

SQUIRE. L. R., \& KNOWLTON, B. J. (1995). Memory, hippocampus, and brain systems. In M. S. Gazzaniga (Ed.), The cognitive neurosciences (pp. 825-837). Cambridge, MA: MIT Press.

SQuire, L. R., \& Zola-Morgan, S. (1991). The medial temporal lobe memory system. Science, 253, 1380-1386.

Tellegen, A.. Lykken, D. T., Bolichard, T. J., Whicox, K. J., Segal, N. L., \& RiCH, S. (1988). Personality similarity in twins reared apart and together. Journal of Personality \& Social Psychology, 54. 1031-1039.

Ursin, H., BaAde, E., \& Levine, S. (Eds.) (1978). Psychobiology of stress: A study of coping men. New York: Academic Press.

VAN DER Kolk, B. A. (1996a). The body keeps the score: Approaches to the psychobiology of posttraumatic stress disorder. In B. A. van der Kolk, A. C. McFarlane, \& L. Weisaeth (Eds.), Traumatic stress: The effects of overwhelming experience on mind, body, and society (pp. 214 241). New York: Guilford.

VAN DER KOLK, B. A. (1996b). The complexity of adaptation to trauma self-regulation, stimulus discrimination, and characterological development. In B. A. van der Kolk, A. C. McFarlane, \& L. Weisaeth (Eds.), Traumatic stress: The effects of overwhelming experience on mind, body, and society (pp. 182-213). New York: Guilford.

VAN DER Kolk, B. A. (1996c). Trauma and memory. In B. A. van der Kolk, A. C. McFarlane, \& L. Weisaeth (Eds.), Traumatic stress: The effects of overwhelming experience on mind, body, and society (pp. 279-302). New York: Guilford.

van der Kolk. B. A., \& MCFarlane, A. C. (1996). The black hole of trauma. In B. A. van der Kolk, A. C. McFarlane, \& L. Weisaeth (Eds.), Traumatic stress: The effects of overwhelming experience on mind body, and society (pp. 3-23). New York: Guilford.

VAN DER KOLK, B. A., \& VAN DER HART, O. (1989). Pierre Janet and the breakdown of adaptation in psychological trauma. American Journal of Psychiatry, 146, 1530-1540.

van der Kol.k, B. A., van der Hart, O., \& Marmar, C. R. (1996). Dissociation and information processing in posttraumatic stress disorder. In B. A. van der Kolk, A. C. McFarlane, \& L. Weisaeth (Eds.) Traumatic stress: The effects of overwhelming experience on mind. body, and society (pp. 303-327). New York: Guilford.

van der Kolk, B. A., Weisaeth. L., \& van der Hart, O. (1996). History of trauma in psychiatry. In B. A. van der Kolk. A. C. McFarlane, \& L. Weisaeth (Eds.), Traumatic stress: The effects of overwhelming experience on mind, bodv, and societv (pp. 47-74). New York: Guilford. 
YeHUdA, R., \& SAPOLSKy, R. M. (1997). Stress and glucocorticoid. Science, 275, 1662-1663.

Yehuda, R., Teicher, M. H., Trestman, R. L., Levengood, R. A., \& SIEver, L. J. (1996). Cortisol regulation in posttraumatic stress disorder and major depression: A chronobiological analysis. Biological Psychiatry, 40, 79-88.

Zola-Morgan, S., \& SQuire, L. R. (1990). The primate hippocampal formation: Evidence for a time-limited role in memory storage. Science, 250, 288-289.

\section{NOTES}

1. The ratio $E_{\text {act }} / R T$ is activation energy expressed as the number of molar energy quanta $R T$ to become dimensionless quantity.

2. Mineralocorticoid receptors have higher affinity to cortisol than do glucocorticoid receptors, so the former predominantly regulate the basal cortisol level, and the latter regulate stress cortisol level (Roozendaal, Cahill, \& McGaugh, 1996).

3. The difference in plasma and urine cortisol levels in these two categories of patients (higher in depression and lower in PTSD) is due to opposite disorders of regulating mechanisms, but hypersecretion is characteristic of both (Goenjian et al., 1996; Yehuda et al., 1996).

4. Although the role of catecholamines (epinephrine) in memorizing is often emphasized, the experiments cited by Korneyev (1997) show that the action of catecholamines is mediated by glucocorticoids. Adrenalectomy decreases learning abilities, whereas administration of glucocorticoids immediately after the training session reverses its effects.

(Manuscript received March 15, 1999;

revision accepted for publication January 18, 2000.) 